\title{
Assembly, Morphology and Thermal Stability of Polyamide 6 Nanotubes
}

\author{
Jian-Jiang LI, Guo-Jun Song, ${ }^{\dagger}$ Xi-Lin She, Ping HAN, Zhi Peng, and Dong CheN \\ Institute of Polymer Materials, Qingdao University, Qingdao 266071, China
}

(Received September 26, 2005; Accepted January 31, 2006; Published May 17, 2006)

\begin{abstract}
Polyamide 6 (PA6) nanotubes array has been prepared successfully using small pore anodic aluminum oxide (AAO) membrane $(200 \mathrm{~nm})$ as the template via polymer solution and melt wetting method. The investigation of Scanning Electron Microscopy (SEM) and Transmission Electron Microscopy (TEM) showed that morphology of polymer nanotubes was highly influenced both by the concentration and the quantity of polymer solution. The Thermal Gravity Analysis (TGA) results indicate that the thermal decomposition temperature of PA6 nanotubes is lower $100^{\circ} \mathrm{C}$ than that of bulk PA6 polymer. However, the thermal decomposition temperature of PA6 nanotubes embedded in AAO membrane is higher than that of bulk polymer. [doi:10.1295/polymj.PJ2005137]

KEY WORDS Polymer Nanotubes / Nanowires / Morphology / Thermal Stability /
\end{abstract}

Nowadays, as a new branch of one-dimensional nanomaterials, polymer nanotubes and nanowires have attracted considerable attention due to their unique properties on account of finite small size and potential applications in a variety of areas. ${ }^{1-5}$ But few researches on polyamide nanotubes have been published so far. As for the preparation of polymer nanotubes, a lot of researches have affirmed that template wetting is a simple and very effective method and some results have been reported by SCIENCE. However, there are many shortages in the previous researches. Firstly, up to now, most of the researches focus on the preparation of conducting polymer nanotubes by chemical synthesis method, ${ }^{6-11}$ Unfortunately, the conducting polymer is easy to be oxidized, and the chemical synthesis method needs rigorous conditions and the reaction is difficult to control. Secondly, although some researchers have reported preparing ordinary polymer nanotubes, such as, PS, ${ }^{12}$ PMMA, ${ }^{13}$ PVDF,${ }^{14}$ etc, which are much stable in air comparing with conducting polymers, but the pore diameter of the templates adopted in those experiments is larger than $500 \mathrm{~nm}$, which is too large as for a size and cannot be obtained commercially. In order to overcome these disadvantages, in this paper, nanotube array of commercial polyamide 6 (PA6) has been prepared firstly using a commercial Anodic Aluminum Oxide (AAO) template with only $200 \mathrm{~nm}$ pore diameter via a simple physical wetting technology. The effects of the concentration and the quantity of polymer solution on the morphology of polymer nanotubes have been investigated by SEM and TEM and the thermal stability of PA6 nanotubes is also studied by TGA.

\section{EXPERIMENTAL}

Anodic aluminum oxide (AAO) membrane with $200 \mathrm{~nm}$ pore diameter was purchased from Whatman Co. Prior to using it, the surface of AAO was cleaned by a ultrasonic cleaner.

\section{Solution Wetting Method}

PA6 was firstly solved in formic acid to prepare 2.5 wt $\%$ and $5.0 \mathrm{wt} \%$ solutions. Firstly, a little amount of solution was put on the top surface of a clean slide glass. And the AAO membrane was quickly put on the solution and taken down after all the solvent evaporated. The membrane was fixed on a slide glass with double-face glue, and then the surface of the membrane was polished using fine grit sandpaper to remove any polymeric surface layer. The sample was then placed in $3 \mathrm{M} \mathrm{NaOH}$ to dissolve the alumina template. The residual membrane was cleaned several times with distilled water and dried at room temperature. Then the sample was sputtered with $\sim 10 \mathrm{~nm}$ of Au prior to SEM (JEOL JSM-840) imaging.

\section{Melt Wetting Method}

PA6 pellet was placed on a controllable hot plate and heated to $200{ }^{\circ} \mathrm{C}$ or $260^{\circ} \mathrm{C}$, respectively. The AAO membrane was then placed on the PA6 melt for $10 \mathrm{~s}$ to wet the wall of the template pore. The subsequent procedure is similar to 2.1. And then the sample was characterized by TEM (JEOL JEM2000EXII).

\section{TGA Measurements}

All the TGA (DTG-50, SHIMADZU) measure-

${ }^{\dagger}$ To whom correspondence should be addressed (Tel: +86-0532-85953260, Fax: +86-0532-85953260, E-mail: songguojun@qdenc.com). 
ments under the following conditions: $\mathrm{N}_{2}$ atmosphere $(50 \mathrm{~mL} / \mathrm{min})$ with a heating rate of $10^{\circ} \mathrm{C} / \mathrm{min}$.

\section{RESULTS AND DISCUSSION}

As we all known, when either a polymer melt or solution is placed on a substrate with high surface energy, it will spread to form a thin film, known as precursor film, similar to the behavior of low molar mass liquids. Similar wetting phenomena occur if porous templates are brought into contact with polymer solution or melts: a thin surface film will cover the pore walls in the initial stages of wetting. This is because the cohesive driving forces for complete filling are much weaker than the adhesive forces. Wall wetting and complete filling of the pores thus take place on different time scales. The latter is prevented by thermal quenching in the case of melts or by solvent evaporation in the case of solutions thus preserving a nanotube structure. ${ }^{15,16}$ The scheme of preparing of polymer nanotube is shown in Figure 1.

Based on the above mechanism, we prepared PA6 nanotubes array and found that the concentration and the quantity of polymer solution should be the main influence factors for the morphology of nanostructures (nanotubes or nanowires). They are discussed as follows.

\section{Preparation of Different Morphology of Nanotube Arrays}

The concentration of solution can affect the morphology of polymer nanotube obviously. The SEM and TEM results of PA6 nanotube array prepared from $2.5 \mathrm{wt} \%$ and $5.0 \mathrm{wt} \%$ formic acid solution are shown in Figure 2.

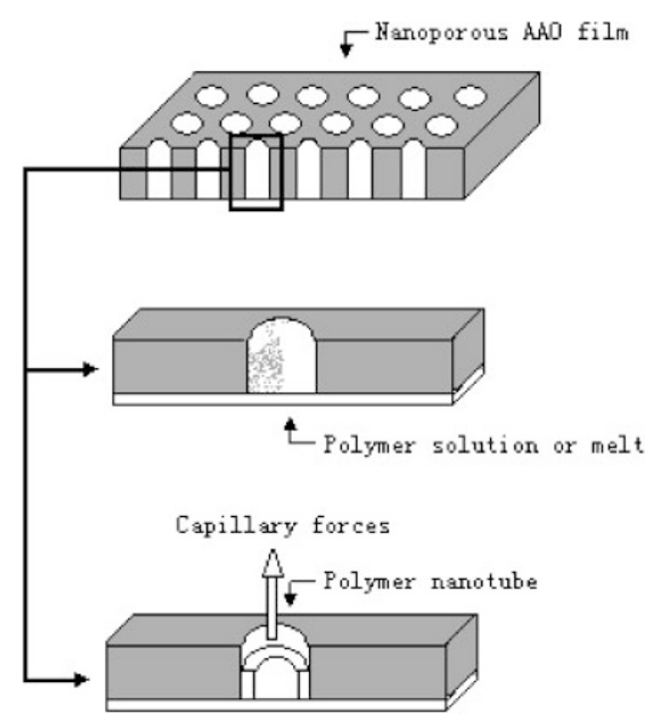

Figure 1. Scheme for preparing polymer nanotubes with AAO template.
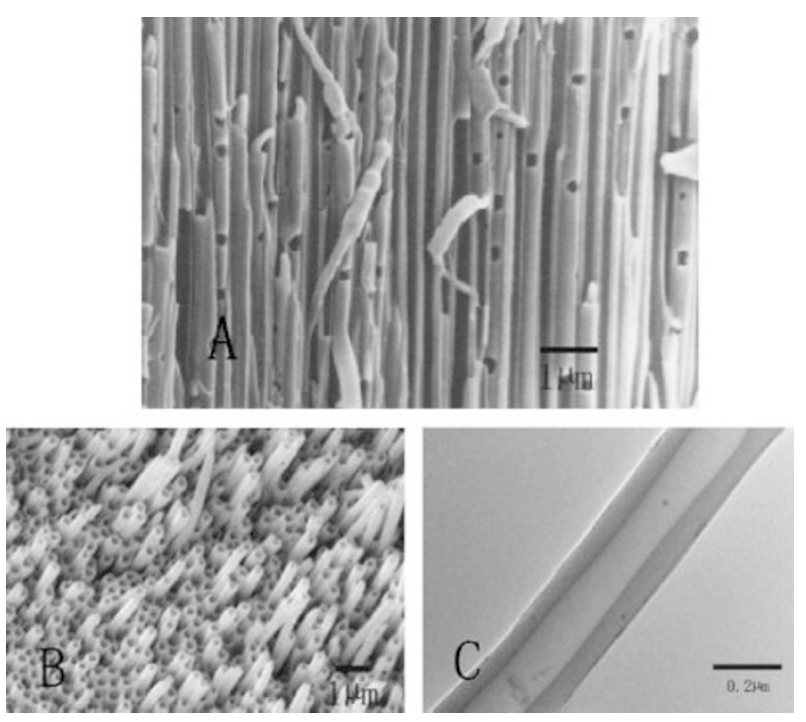

Figure 2. SEM and TEM of PA6 nanotubes. A. PA6 nanotube array prepared from a $2.5 \mathrm{wt} \%$ formic acid solution. B. PA6 nanotube array prepared from a $5.0 \mathrm{wt} \%$ formic acid solution. C. TEM of PA6 nanotube gained from Figure 2B.

PA6 nanotubes prepared from a $2.5 \mathrm{wt} \%$ polymer solution is shown in Figure 2A. It depicts that many PA6 nanotubes are embedded in the channel of AAO membrane. We may find that almost all the PA6 nanotubes have pinholes and gaps. These kinds of defections may be caused by the low concentration of polymer solution where there are not enough polymer chains to deposit within the template pores. In order to confirm the above assumption, another experiments are executed. PA6 nanotube array prepared from a $5.0 \mathrm{wt} \%$ solution is shown in Figure 2B. It can be seen that the top ends of all the nanotubes are open and have smooth surface and also have uniform outer diameter. It also can be found that the microscopic appearance of Figure 2B array is regular and without defection compared with Figure 2A. The result proves that the concentration of polymer solution is an important factor to the morphology of the PA6 nanotube array.

Figure 2C shows the TEM of a single PA6 nanotube prepared from $5.0 \mathrm{wt} \%$ solution. The nanotube is hollow. Its wall thickness is $c a .40 \mathrm{~nm}$ and outer diameter is $c a .200 \mathrm{~nm}$ that is in accord with the pore diameter of AAO membrane. This fact implies that there are strong interaction between the polymer chains and the pore walls. The polymer nanotubes should be formed under the inducement of the template pore.

\section{Preparation of Different Length of Nanotubes}

The preparation of different length of polymer nanotubes is important for its further processing in manufacturing nanodevices. And the length can be 

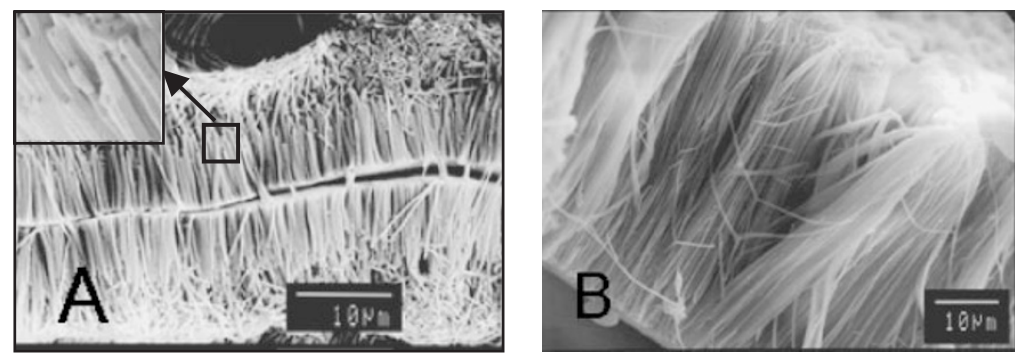

Figure 3. SEM of different length of PA6 nanotube array prepared by $1 \mu \mathrm{L}$ (Figure 3A) and $5 \mu \mathrm{L}$ (Figure $3 \mathrm{~B}$ ) of a $2.5 \mathrm{wt} \%$ polymer solution, respectively.

controlled by using different quantity of polymer solution in the wetting procedures. The results are shown in Figure 3.

In this experiment, the round $\mathrm{AAO}$ membrane has an area of $133 \mathrm{~mm}^{2}$ and thickness of $60 \mu \mathrm{m}$. Figure 3A shows the side-view of PA6 nanotube array and the quantity of polymer solution used in the preparation is $1 \mu \mathrm{L}$. It can be seen that the length of nanotubes is $c a .10 \mu \mathrm{m}$, which is much shorter than the entire thickness of AAO membrane and all the nanotubes are hollow (as shown in the amplified picture in Figure 3A) because the polymer solution hasn't run through the entire pores.

Figure 3B shows the side-view of PA6 nanotubes array and the quantity of polymer solution used in the preparation is $5 \mu \mathrm{L}$. It can be seen that the length of PA6 nanotubes is $c a .60 \mu \mathrm{m}$, which is almost same to the length of the template pores. It indicates that the polymer solution has run through the entire AAO membrane. In this condition, most of the tips of the nanotubes are covered because the redundant solution forms a thin polymeric layer on the membrane's upper surface. In a word, different length of nanotube array can be obtained via control the quantity of polymer solution and the quantity must be lower than $5 \mu \mathrm{L}$ in order to obtain open-mouth PA6 nanotubes.

\section{Preparation of Nanotube Array Using Melts Wetting Method}

PA6 nanotubes can also be prepared by melt wetting method. The SEM of PA6 nanotubes obtained from this method shown in Figure 4.

The PA6 nanotube array prepared at $200^{\circ} \mathrm{C}$ is shown in Figure 4. The nanotubes are hollow with uniform diameter and regular array. The wall thickness of the nanotubes is $c a .30 \mathrm{~nm}$ and the outer diameter is $c a$. $200 \mathrm{~nm}$ that is accordant to the pore diameter of AAO membrane. It is clear that PA6 melt also has excellent ability to form nanotubes. But the wall thickness is thinner than that of the PA6 nanotubes prepared from solution wetting method. As we all known, the viscidity of melt is higher than that of polymer solution. So the liquidity of melt is poor than that of polymer solu-

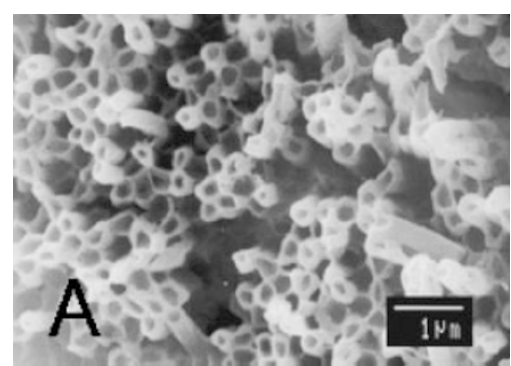

Figure 4. SEM of PA6 nanotubes prepared from PA6 melt at $200^{\circ} \mathrm{C}$.

tion, and so it is difficult to wet the membrane comparing with the polymer solution. This is the reason why the wall thickness prepared from melt wetting method is thinner than that of the PA6 nanotubes prepared from solution wetting method.

\section{Thermal Stability of PA6 Nanotubes}

Nanomaterials always have special properties because of their small size effects. And some researches indicate that the thermal decomposition temperature of polymer nanotube is higher than that of bulk polymer. For instance, Shilun Qiu ${ }^{17}$ has reported that the thermal decomposition of Polystyrene nanotubes is higher $150^{\circ} \mathrm{C}$ than the bulk polystyrene at least. We investigated the PA6 nanotubes by TGA and discussed it as follows.

Curve 1 shown in Figure 5 is the TGA curve of bulk PA6 polymer. It can be sure that the decomposition temperature of bulk PA6 polymer is ranging from $400-500^{\circ} \mathrm{C}$ and the temperature of maximum decomposition rate is $460^{\circ} \mathrm{C}$. Curve 2 shown in Figure 5 is the TGA curve of PA6 film prepared from PA6 solution. We may find that bulk PA6 polymer and PA6 film have the same shape TGA curve. The fact indicates that bulk PA6 polymer and PA6 film have similar thermal properties.

Curve 3 shown in Figure 5 is the TGA curve of pure PA6 nanotubes. It can be seen that its decomposition Temperature is ranging from $300-400{ }^{\circ} \mathrm{C}$. Its maximum decomposition rate happened at $360^{\circ} \mathrm{C}$ and most of the weight of the nanotubes has lost 


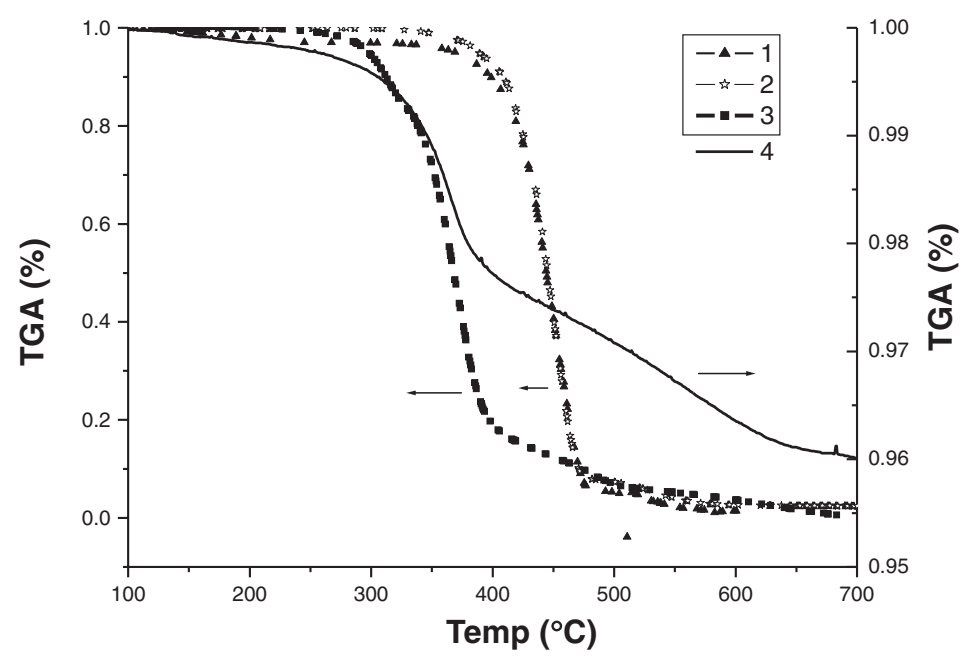

Figure 5. TGA curves of different morphologies of PA6 1-Pure PA6; 2-PA6 film; 3-PA6 Nanotubes; 4-Powder of PA6 Nanotubes embedded in nanoporous of AAO Membrane.

before $400{ }^{\circ} \mathrm{C}$. In addition, there is only a little losing weight from $400-500^{\circ} \mathrm{C}$. The little losing weight is caused by the PA6 film attached on the end of the nanotubes because its decomposition temperature is the same to the PA6 film. So we are sure that the decomposition temperature of pure PA6 nanotubes is lower $100^{\circ} \mathrm{C}$ than that of bulk PA6 polymer.

Curve 4 shown in Figure 5 is the TGA curve of the powder of PA6 nanotubes embedded in the nanoporous of AAO membrane. From it, we may find that the thermal degradation happened at $360{ }^{\circ} \mathrm{C}$ is similar to the curve 3 for PA6 nanotubes. It may be caused by the PA6 nanotubes exposed out of the nanoporous of AAO membrane after rubbing. After rubbing, some PA6 nanotubes are still embedded in the nanoporous of AAO membrane. And the thermal degradation took place at $550^{\circ} \mathrm{C}$ may be caused by this portion of PA6 nanotubes. It can be seen that the decomposition temperature has an obvious delay comparing with curve 3 . The fact indicates that the AAO membrane could delay the decomposition of PA6 nanotubes. The phenomena may be caused by the strong interaction between the PA6 nanotubes and the inner wall of nanoporous. It needs further research to supply other more reasonable explanations.

\section{CONCLUSIONS}

We have successfully prepared PA6 polymer nanotubes via solution and melt wetting method using relatively small diameter pore AAO membrane as the template. The method is simple and effective for preparing polymer nanotube array and controlling the morphology of the nanotubes. The TGA results indicate that the thermal decomposition temperature of PA6 nanotubes is lower $100{ }^{\circ} \mathrm{C}$ than that of bulk
PA6 polymer. However, the thermal decomposition temperature of PA6 nanotubes embedded in AAO membrane is higher than that of bulk polymer. This PA6 nanotube array could be regarded as "secondtemplate" to prepare coaxial nanocables via filling other materials into the inner space of nanotubes. And it also could be made into functional nanodevices via chemical modifying the inner wall of the nanotubes.

Acknowledgment. This project was supported by the National Natural Science Foundation of China. (No. 50473012)

\section{REFERENCES}

1. M. Steinhart, J. H. Wendorff, and A. Greiner, et al., Science, 296, 1997 (2002).

2. E. P. S. Tan and C. T. Lim, Appl. Phys. Lett., 84, 1603 (2004).

3. S. Ai, G. Lu, Q. He, and J. Li, J. Am. Chem. Soc., 125, 11140 (2003).

4. S. Cuenot, S. Demoustier-Champagne, and B. Nysten, Phys. Rev. Lett., 85, 1690 (2000).

5. L. Danginet-De Pra and S. Demoustier-Champagne, Polymer, 46, 1583 (2005).

6. M. Mazur, M. Tagowska, B. Palys, and K. Jackowska, Electrochem. Commun., 5, 403 (2003).

7. T. Shimomura, T. Akai, T. Abe, and K. 1to, J. Chem. Phys., 116, 1753 (2002).

8. S. K. Saha, Appl. Phys. Lett., 81, 3645 (2002).

9. Z.-J. Wei and M.-X. Wan, Adv. Mater., 14, 1314 (2002).

10. J. Joo, B. H. Kim, D. H. Park, and H. S. Kim, etc., Synth. Met., 153, 313 (2005).

11. B. H. Kim, D. H. Park, J. Joo, S. G. Yu, and S. H. Lee, Synth. Met., 150, 279 (2005).

12. G.-J. Song, X.-L. She, and J.-J. Li, et al., J. Mater. Res., 19, 3324 (2004). 
13. V. M. Cepak, and C. R. Martin, Chem. Mater., 11, 1363 (1999).

14. M. Steinhart, S. Senz, and R. B. Wehrspohn, and J. H. Wendorff, Macromolecules, 36, 3646 (2003).

15. P. G. de Gennes, Rev. Mod. Phys., 57, 827 (1985).
16. “Surfactant Science,” S. F. Kistler, J. C. Berg, Ed., Marcel Dekker, New York, 1993, chap. 6, p 49.

17. B.-S. Li, G.-S. Zhu, and F.-F. Cao, et al., Polymer Prepr. (Am. Chem. Soc., Div. Polym. Chem.), 43(2), 414 (2002). 